OPEN ACCESS

Edited by:

Roumen Kirov,

Institute of Neurobiology (BAS),

Bulgaria

Reviewed by:

Bong Ju Lee,

Inje University Haeundae Paik Hospital, South Korea

Satoru Ikezawa

Japan Agency for Medical Research and Development, Japan

${ }^{*}$ Correspondence: Yoshie Omachi yomachi@ncnp.go.jp

Specialty section

This article was submitted to

Psychopathology, a section of the journal Frontiers in Psychiatry

Received: 08 January 2018

Accepted: 29 August 2018

Published: 20 September 2018

Citation:

Omachi Y and Sumiyoshi T (2018) Dose Reduction/Discontinuation of Antipsychotic Drugs in Psychosis; Effect on Cognition and Functional Outcomes. Front. Psychiatry 9:447. doi: 10.3389/fpsyt.2018.00447

\section{Dose Reduction/Discontinuation of Antipsychotic Drugs in Psychosis; Effect on Cognition and Functional Outcomes}

\author{
Yoshie Omachi ${ }^{1 *}$ and Tomiki Sumiyoshi ${ }^{2}$ \\ ${ }^{1}$ Department of Psychiatry, National Center Hospital, National Center of Neurology and Psychiatry, Tokyo, Japan, \\ ${ }^{2}$ Department of Preventive Intervention for Psychiatric Disorders, National Institute of Mental Health, National Center of \\ Neurology and Psychiatry, Tokyo, Japan
}

Backgrounds: There is a debate regarding the optimal timing of discontinuation of antipsychotic drugs in patients with first episode psychosis (FEP) or schizophrenia. We aimed to provide a review of the literature on which strategy (medication maintenance vs. dose reduction/discontinuation) is more likely to maximize outcomes, such as cognition and social function.

Methods: Using PubMed, the Cochrane Library and systematic reviews, articles published between 2007 and 2018 were reviewed, which investigated the effect of dose reduction/discontinuation vs. maintenance treatment on measures of cognition and/or social function in FEP and schizophrenia.

Results: Six studies were identified; 2 studies reported on cognition while 4 studies concern social function. All studies except one reported that improvement of functional outcomes in remitted patients with FEP or schizophrenia allocated to a dose reduction/discontinuation arm was equal to or better than that in patients for whom medication doses were maintained. One trial of social function with a 1-year follow-up period found a greater improvement in the medication maintenance group, while no group difference was observed with 3-year and 10-year follow-up periods. On the other hand, a 7-year follow-up study found a superiority for the dose reduction/discontinuation regimen in terms of social outcome. Two studies on cognition with a short follow-up period reported a greater improvement for the dose reduction/discontinuation group.

Conclusions: Information on cognition and social function has been relatively sparse. These measures of functional outcome should be considered in deciding which strategy of antipsychotic treatments is beneficial in individual cases with FEP or schizophrenia.

Keywords: first episode psychosis, schizophrenia, discontinuation, maintenance, antipsychotics, cognition, social function, functional outcome 


\section{INTRODUCTION}

There is controversy about the continued use of antipsychotic drugs in patients with psychotic disorders, including schizophrenia. For example, treatment guidelines for first episode psychosis (FEP) recommend at least 1-year of antipsychotic treatment following remission (1). Antipsychotic medication may affect relapse and remission rates over time. In fact, randomized controlled trials (RCTs) have reported a considerably high relapse rate after dose reduction/discontinuation of antipsychotic treatment (2-5). From the clinical point of view, the maintenance/discontinuation debate should also encompass other aspects, such as functional outcome.

Functional outcome consists of several domains, such as psychosocial skill acquisition, instrument skills/social problemsolving ability, and community outcome/daily activities (6). A general consensus is that recovery in psychosis contains achievements of a personally acceptable quality of life and feeling of self-esteem (7). These outcome measures have been suggested to receive a higher priority than symptom management for young people with psychosis (8). Specifically, there is convincing evidence for an association between cognition and functional recovery in schizophrenia, as cognition may provide a better correlate of functional outcome than psychotic symptoms $(6,9)$. Accordingly, Sumiyoshi et al. (10) reported temporal associations between cognition and social function in patients with schizophrenia. Longitudinally, Fu et al. (11) found that performance on tests of attention, verbal learning, and verbal working memory are associated with social function throughout a 4-year observation period. Consequently, efforts have been made to develop therapeutics for disturbances of cognition (10).

To date, effects of antipsychotic drugs on cognition in patients with schizophrenia have been intensively examined. Initially, the second-generation antipsychotic drugs (SGAs) were suggested to ameliorate cognitive impairment more effectively than the first-generation antipsychotic drugs (FGAs). However, results from recent meta-analyses of RCTs on the effect of SGAs vs. FGAs, or SGAs vs. placebo indicate that only clozapine elicits cognitive benefits in patients with schizophrenia (12-14). So far, no consensus has been established as to an appropriate duration of antipsychotic treatments, particularly with regard to cognitive and social outcomes.

Some clinicians may feel that the quality of life (QOL) in patients with FEP would be better if antipsychotic medications are discontinued following remission (15). To our knowledge, there has been little information on the effects of maintenance vs. dose reduction/discontinuation of antipsychotic treatments on cognitive and social function in patients with FEP or schizophrenia. Therefore, the aim of this article is to provide an overview on which strategy is more beneficial for these outcomes.

\section{MATERIALS AND METHODS}

\section{Search Strategy and Selection Criteria}

We searched PubMed, the Cochrane Library, and systematic reviews for randomized and non-randomized controlled trials published between 2007 and 2018, using the following search string; (schizophrenia OR psychosis OR first episode) AND (dose reduction OR discontinuation) AND (cognition OR social OR function OR relapse OR remission OR recovery). We also searched the reference lists of previous reviews, which compared the effects of medication maintenance vs. dose reduction/discontinuation on relapse, remission, cognition, and/or social function in FEP or schizophrenia (16-19). The searches were limited to English language articles and titles/abstracts. Due to the limited number of RCTs on functional outcome between the two groups (dose reduction/discontinuation vs. maintenance) in FEP, we included open-label randomized controlled trials and non-randomized prospective studies.

\section{RESULTS}

\section{Study Design}

We have identified five open-label and one double-blind studies comparing cognitive and/or social functional consequences between maintenance and dose reduction/discontinuation of antipsychotic treatments in patients with FEP or schizophrenia. Out of them, five were RCTs $(3,4,20-23)$, whereas one was a non-randomized, prospective study (24). A summary of these studies, including the methodology and results, is presented in Table 1 (for comparisons between studies, relapse rates were calculated, where necessary, by diving the number of patients who relapsed during observation periods by the total number of patients in the corresponding group). Subjects included patients with schizophrenia, schizophrenia spectrum disorders, or FEP, with various regimens in terms of medication dose reduction/discontinuation, follow-up period, and outcome measures. Sample sizes ranged from 42 to 178 , and the length of follow-up ranged from 28 weeks to 10 -years.

\section{Outcome Measures}

Two studies included measures of cognition, while 4 studies used measures of social function at follow-up.

Faber et al. (20) used a test battery consisting of the Stroop 2 (color naming) and 3 (color-word naming) tests, continuous performance test (CPT), digit span forward and backward, California Verbal Learning Test, Trail-Making A and $B$, verbal (category) fluency (animals and professions), Symbol Substitution Test, and Finger tapping $(25,26)$. These tests have been shown to represent attention, working memory, verbal memory, cognitive speed of processing, and motor speed.

Takeuchi et al. (22) assessed cognition with the Repeatable Battery for the Assessment of Neuropsychological Status (RBANS) $(27,28)$. This battery yields scaled scores for 5 cognitive domains, i.e., immediate memory, visuospatial/constructional ability, language, attention, and delayed memory.

Gaebel et al. (4) assessed social function with the Global Assessment of Functioning (GAF) (29). This scale measures symptom severity, as well as psychological, social, and occupational functioning during specified periods, on a continuum from mental health (score 100) to mental illness (score 0) (30). QOL was measured by the Lancashire Quality of Life Profile (LQLP) (31). The LQLP focuses on nine specific 


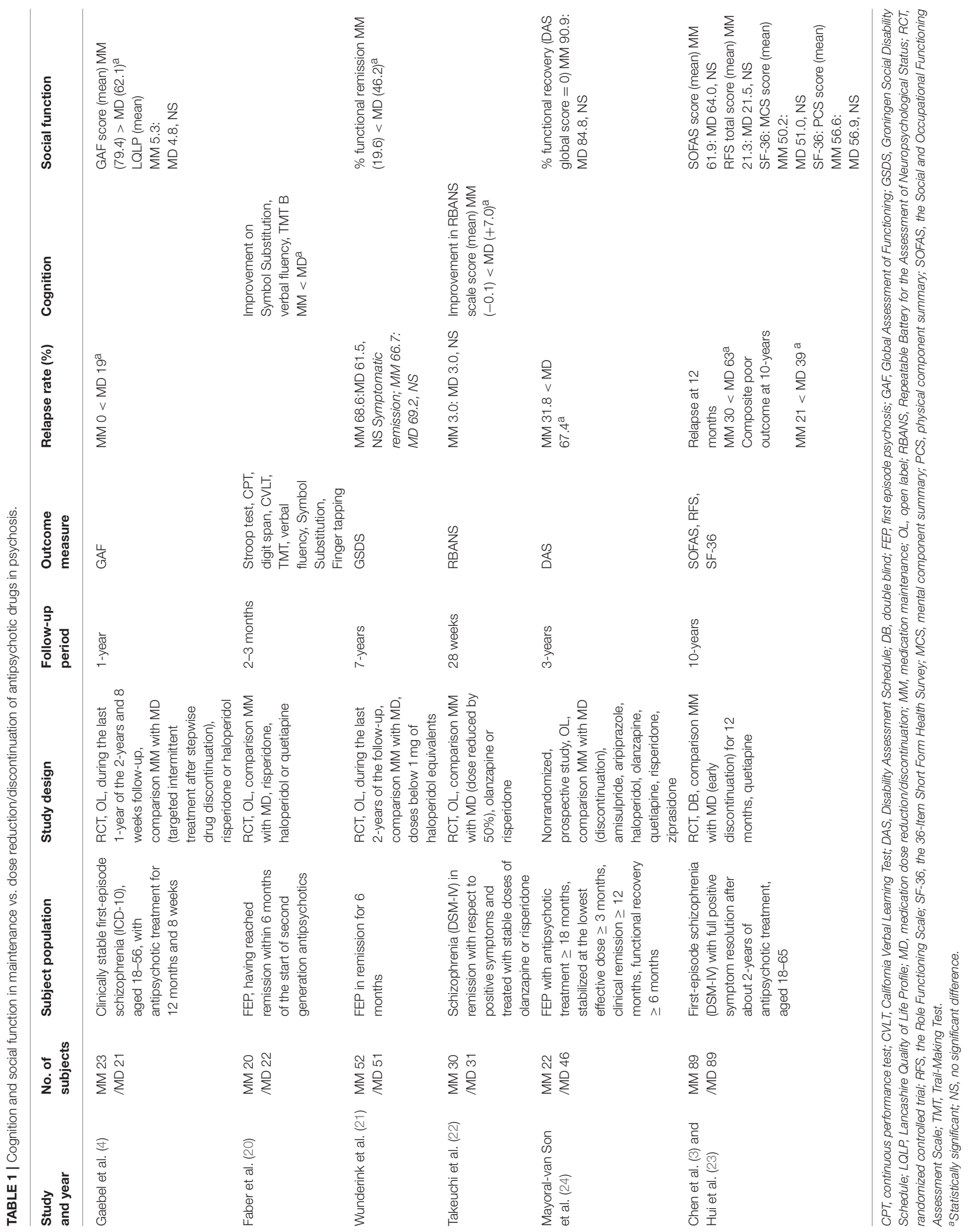


domains; living situation, family, social relationships, leisure activities, work/education, finances, personal safety, health, and religion. The questions pertaining to the subjective QOL appraisal allow patients to rate their satisfaction on a seven-point scale (32).

Wunderink et al. (21) evaluated social function with the Groningen Social Disability Schedule (GSDS) (33), a semistructured investigator-based interview measuring disabilities in social function. Seven items from the GSDS were used; selfcare, housekeeping, family relationship, partner relationship, relationship with peers, community integration, and vocational functioning. A patient with functional remission should function adequately in all 7 domains with none or only a minimal disability in all of them (not allowing a score of 2 or 3 ).

Mayoral-van Son et al. (24) used the global disability item from the Spanish version of the Disability Assessment Schedule (DAS). This item has a score range from 0 (no disability) to 5 (gross disability). They dichotomized functional status into "functional recovery" and "functional deficits." The "functional recovery" status indicates that the patient is currently participating in part-time (paid and fewer than $35 \mathrm{~h}$ per week) or full-time work or study with the same or better level of performance as before the psychotic episode, and has no functional disability (score of 0 in the DAS).

Hui et al. (23) assessed social function with the Social and Occupational Functioning Assessment Scale (SOFAS) and the Role Functioning Scale (RFS), as well as health-related QOL with the 36-Item Short Form Health Survey (SF-36). The SOFAS assesses social and occupational functioning, whose scores are not directly influenced by overall severity of the individual's psychological symptoms $(34,35)$. The scores range from 1 to 100 , with lower scores representing impaired functioning. The RFS measures the functioning level in patients with psychiatric disorders, focusing on four domains; working productivity, independent living and self-care, immediate social network relationships, and extended social network relationships (36). Each domain is rated on a 7-point scale, with lower scores representing lower functioning. The SF-36 consists of 36 questions on functional health and well-being (37), as summarized by two indices, i.e., the mental component summary (MCS) and physical component summary (PCS). The MCS includes four domains; vitality (energetic or fatigued), social functioning, role limitations because of emotional problems, and general mental health (psychological distress and well-being), while the PCS consists of four domains; physical functioning, role limitations because of physical health problems, bodily pain, and general health perceptions. SF-36 scores range from 0 to 100 , with higher scores indicating better functional health and well-being.

\section{Relapse Rate}

In the reviewed studies, relapse rates range from 3.0 to $67.4 \%$ in dose reduction/discontinuation groups, and $0-68.6 \%$ in medication maintenance groups. Three trials $(3,4,24)$ with a follow-up period less than 3-years reported a higher rate of relapse in the dose reduction/discontinuation groups, while a 7 year follow-up study (21) found that relapse rates of the two groups (dose reduction vs. maintenance) were equal. In a 10 year follow-up study (23), the incidence of persistent positive symptoms, requirement for clozapine, or death by suicide occurred in $39 \%$ in the discontinuation group and $21 \%$ in the maintenance group (risk ratio 1.84, 95\% CI 1.15-2.96; $p=0.01$ ).

\section{Cognition}

Faber et al. (20) found that the medication discontinuation group showed a significantly greater improvement than the maintenance group on scores of the Symbol Substitution Test $(F=4.49, d f=1.40, P<0.05)$, verbal fluency task $(F=6.11, d f=1.40, p<0.05)$ and Trail-Making Test$\mathrm{B}(F=5.54, d f=1.40, p<0.05)$ at $2-3$ months after the start of dose reduction/discontinuation. Similarly, Takeuchi et al. (22) reported that the dose reduction group showed a significantly greater improvement in RBANS scores compared with the maintenance group [mean (SD), 7.0 (7.1) vs. -0.1 (8.0), $p<0.001]$.

\section{Social Function}

Gaebel et al. (4) reported that the medication maintenance group showed a greater mean GAF score at 1-year follow-up point than that in the dose reduction/discontinuation group [79.4 (10.1) vs. 62.1 (16.7), $p<0.001]$. On the other hand, no between-group difference was found for QOL [5.3 (1.2) vs. 4.8 (0.9), NS]. By contrast, Wunderink et al. (21) found that the functional remission rate after 7-years was significantly higher for the dose reduction/discontinuation group compared with the maintenance group ( 19.6 vs. $46.2 \%, p<0.01$ ). On the other hand, Mayoral-van Son et al. (24) did not find a difference in the functional status at the 3-year follow-up point between the two groups (90.9 vs. $84.8 \%$, NS). Likewise, Hui et al. (23) did not observe a significant difference in the functional status at the 10year follow-up point between the two groups; [61.9 (9.6) vs. 64.0 (8.9), NS] on SOFAS [21.3 (3.4) vs. 21.5 (2.8), NS] on RFS Total, [50.2 (9.1) vs. 51.0 (8.4), NS] on SF-36 (MCS) and [56.6 (7.6) vs. 56.9 (6.6), NS] on SF-36 (PCS).

\section{DISCUSSION}

The present review identified 2 studies reporting on cognition and 4 studies on social function, which compared functional outcomes between medication maintenance vs. dose reduction/discontinuation patients with FEP or schizophrenia. In spite of abundant information on relapse rates, only the limited number of studies have dealt with these outcomes, particularly, cognition. Of note, all studies except one (4) reported the advantage of the dose reduction/discontinuation method over the maintenance strategy in terms of functional outcomes.

In terms of social function, a trial with a 1-year follow-up period (4) found a greater improvement in the maintenance group, while there was no significant group difference 3-years and 10-years after the start of follow-up $(23,24)$. On the other hand, the study with a 7-year follow-up period (21) observed a superiority for the dose reduction/discontinuation regimen. The difference in study design may account for the discrepant results. 
For example, social function was measured by the GAF in the 1year follow-up study (4). The GAF assesses psychotic symptoms and overall functional ability simultaneously; when symptom severity and level of functioning are discordant, clinicians are directed to use the rating that reflects the lower of the two levels $(30,38)$. In the 1-year follow-up study $(4)$, the high relapse rate in the dose reduction/discontinuation group may have affected symptom severity, leading to worsening of GAF scores (assumed to represent "function" status). The use of standardized scale to assess real-world social function, independent of symptom severity, would be desired to circumvent this issue. Regarding the study with a 7 -year follow-up period $(2,21)$, both groups were similar in their interventions, because about $80 \%$ in the dose reduction/discontinuation group failed to discontinue drug treatment (39). In addition, follow-up was generally naturalistic and unblinded, so there could be a difference in unmeasured psychosocial aspects, such as community care and number of visits (39). Furthermore, the differences in diagnostic categories in the 2 groups may be a plausible explanation toward the significantly better recovery and functional remission in the dose reduction/discontinuation group (40). Further study is warranted to determine the effect of length of observation periods on social function in patients who (dis)continue medications.

A greater improvement in cognition for the dose reduction/discontinuation group was reported in two studies $(20,22)$ with a relatively short follow-up period of less than 3 months. Fu et al. (11) found that performance on tests of attention and verbal working memory predicted social function, as measured by the Global Functioning (Social and Role) (11), in addition to temporal associations between social function vs. attention, verbal working memory, and verbal learning memory at baseline. Also, a longer observation period revealed that a greater cumulative lifetime antipsychotic use led to poorer cognitive performance in later life in patients with schizophrenia, which may be caused by disorganization symptoms (41). Future studies should consider type of cognitive domains and potential effects of key clinical features on cognitive and functional outcomes.

Part of the results from this review suggests that dose reduction/discontinuation of antipsychotic treatments after remission leads to better functional outcome in FEP and schizophrenia. In addition, Wunderink et al. (21) reported that milder negative symptoms, living together, and better social function at baseline are associated with better functional outcomes. Meanwhile, we often experience chronic patients exhibiting severe functional impairment as a result of repeated relapses after discontinuation of antipsychotic drugs. In fact, Mayoral-van Son et al. (24) found that relapsed patients showed

\section{REFERENCES}

1. Group IEPAW. International clinical practice guidelines for early psychosis. Br J Psychiatry Suppl. (2005) 48:s120-4. doi: 10.1192/bjp.187.48.s120

2. Wunderink L, Nienhuis FJ, Sytema S, Slooff CJ, Knegtering R, Wiersma D. Guided discontinuation versus maintenance treatment in remitted more severe symptoms and poorer functional status at the end of follow-up periods. Further, Gaebel et al. (42) found that social function was significantly poorer in patients who relapsed after drug discontinuation compared to those without relapse. These observations indicate a need for the search of predicting factors to identify patients who need continued medications.

Previous studies have attempted to identify patients who will be benefitted or jeopardized by dose reduction/discontinuation of antipsychotic drugs. Accordingly, Wunderink et al. (21) found that a short duration of untreated psychosis was the strongly associated with achievements of symptom remission. Moreover, Alvarez-Jimenez et al. (16) noted that risk factors of relapse after discontinuation or dose reduction include diagnosis of schizophrenia, longer duration of illness, and poor pre-morbid functioning. They also reported that psychosocial interventions for FEP coupled with antipsychotic drugs were effective in preventing relapses (43). Furthermore, the guidelines devised by the International Early Psychosis Association (1) recommend that the minimal dose antipsychotic medication should be continued for preventing relapse and impairment of functions in FEP patients with risks of relapse.

In conclusion, although dose reduction/discontinuation of antipsychotic medication may be associated with higher relapse rates, this strategy may improve cognitive outcomes in some patients with FEP or schizophrenia. In this line, predictors for successful dose reduction/discontinuation deserve further explorations. So far, information on cognition and social function is relatively sparse. These measures of functional outcome should be considered in deciding which strategy of antipsychotic treatments is encouraged in individual cases.

\section{AUTHOR CONTRIBUTIONS}

TS planned and initiated this work. YO performed the literature search and drafted the first manuscript. Both authors revised the manuscript and approved the final version.

\section{FUNDING}

Part of this work was supported by Japan Society for the Promotion of Science KAKENHI Grant Number No. 17K10321, Intramural Research Grant (29-1, 30-1, 30-8) for Neurological and Psychiatric Disorders of NCNP.

\section{ACKNOWLEDGMENTS}

We acknowledge discussions with Dr. Kazuyuki Nakagome. 
4. Gaebel W, Riesbeck M, Wölwer W, Klimke A, Eickhoff M, von Wilmsdorff $\mathrm{M}$, et al. Relapse prevention in first-episode schizophreniamaintenance vs intermittent drug treatment with prodrome-based early intervention: results of a randomized controlled trial within the German Research Network on Schizophrenia. J Clin Psychiatry (2011) 72:205-18. doi: 10.4088/JCP.09m05459yel

5. Landolt K, Rössler W, Ajdacic-Gross V, Derks EM, Libiger J, Kahn RS, et al. Predictors of discontinuation of antipsychotic medication and subsequent outcomes in the European First Episode Schizophrenia Trial (EUFEST). Schizophr Res. (2016) 172:145-51. doi: 10.1016/j.schres.2016. 01.046

6. Green MF, Kern RS, Braff DL, Mintz J. Neurocognitive deficits and functional outcome in schizophrenia: are we measuring the "right stuff"? Schizophr Bull. (2000) 26:119-36. doi: 10.1093/oxfordjournals.schbul.a0 33430

7. Law H, Morrison AP. Recovery in psychosis: a Delphi study with experts by experience. Schizophr Bull. (2014) 40:1347-55. doi: 10.1093/schbul/sbu047

8. Iyer SN, Mangala R, Anitha J, Thara R, Malla AK. An examination of patientidentified goals for treatment in a first-episode programme in Chennai, India. Early Interv Psychiatry (2011) 5:360-5. doi: 10.1111/j.1751-7893.2011.0 0289.x

9. Green MF, Harvey PD. Cognition in schizophrenia: past, present, and future. Schizophr Res Cogn. (2014) 1:e1-9. doi: 10.1016/j.scog.2014. 02.001

10. Sumiyoshi T, Nishida K, Niimura H, Toyomaki A, Morimoto T, Tani M, et al. Cognitive insight and functional outcome in schizophrenia; a multicenter collaborative study with the specific level of functioning scale-Japanese version. Schizophr Res Cogn. (2016) 6:9-14. doi: 10.1016/j.scog.2016.08.001

11. Fu S, Czajkowski N, Rund BR, Torgalsbøen AK. The relationship between level of cognitive impairments and functional outcome trajectories in first-episode schizophrenia. Schizophr Res. (2017) 190:144-9. doi: 10.1016/j.schres.2017.03.002

12. Takeuchi H, Thiyanavadivel S, Fervaha G, Remington G. Neurocognitive benefits of second-generation antipsychotics versus placebo: insufficient evidence based on a systematic review. J Clin Psychopharmacol. (2017) 37:2746. doi: 10.1097/JCP.0000000000000662

13. Nielsen RE, Levander S, Kjaersdam Telléus G, Jensen SO, Østergaard Christensen $T$, Leucht $S$. Second-generation antipsychotic effect on cognition in patients with schizophrenia-a meta-analysis of randomized clinical trials. Acta Psychiatr Scand. (2015) 131:185-96. doi: 10.1111/acps. 12374

14. Hill SK, Bishop JR, Palumbo D, Sweeney JA. Effect of second-generation antipsychotics on cognition: current issues and future challenges. Expert Rev Neurother. (2010) 10:43-57. doi: 10.1586/ern.09.143

15. Thompson A, Singh S, Birchwood M. Views of early psychosis clinicians on discontinuation of antipsychotic medication following symptom remission in first episode psychosis. Early Interv Psychiatry (2016) 10:355-61. doi: 10.1111/eip.12244

16. Alvarez-Jimenez M, O’Donoghue B, Thompson A, Gleeson JF, Bendall S, Gonzalez-Blanch C, et al. Beyond clinical remission in first episode psychosis: thoughts on antipsychotic maintenance vs. guided discontinuation in the functional recovery era. CNS Drugs (2016) 30:357-68. doi: 10.1007/s40263-016-0331-x

17. Karson C, Duffy RA, Eramo A, Nylander AG, Offord SJ. Long-term outcomes of antipsychotic treatment in patients with first-episode schizophrenia: a systematic review. Neuropsychiatr Dis Treat. (2016) 12:57-67. doi: 10.2147/NDT.S96392

18. Zhu Y, Krause M, Huhn M, Rothe P, Schneider-Thoma J, Chaimani A, et al. Antipsychotic drugs for the acute treatment of patients with a first episode of schizophrenia: a systematic review with pairwise and network metaanalyses. Lancet Psychiatry (2017) 4:694-705. doi: 10.1016/S2215-0366(17)3 0270-5

19. Zipursky RB, Menezes NM, Streiner DL. Risk of symptom recurrence with medication discontinuation in first-episode psychosis: a systematic review. Schizophr Res. (2014) 152:408-14. doi: 10.1016/j.schres.2013.08.001

20. Faber G, Smid HG, Van Gool AR, Wiersma D, Van Den Bosch RJ. The effects of guided discontinuation of antipsychotics on neurocognition in first onset psychosis. Eur Psychiatry (2012) 27:275-80. doi: 10.1016/j.eurpsy.2011. 02.003

21. Wunderink L, Nieboer RM, Wiersma D, Sytema S, Nienhuis FJ. Recovery in remitted first-episode psychosis at 7 years of follow-up of an early dose reduction/discontinuation or maintenance treatment strategy: long-term follow-up of a 2-year randomized clinical trial. JAMA Psychiatry (2013) 70:913-20. doi: 10.1001/jamapsychiatry.2013.19

22. Takeuchi H, Suzuki T, Remington G, Bies RR, Abe T, Graff-Guerrero A, et al. Effects of risperidone and olanzapine dose reduction on cognitive function in stable patients with schizophrenia: an open-label, randomized, controlled, pilot study. Schizophr Bull. (2013) 39:993-8. doi: 10.1093/schbul/ sbt090

23. Hui CLM, Honer WG, Lee EHM, Chang WC, Chan SKW, Chen ESM, et al. Long-term effects of discontinuation from antipsychotic maintenance following first-episode schizophrenia and related disorders: a 10 year followup of a randomised, double-blind trial. Lancet Psychiatry (2018) 5:432-42. doi: 10.1016/S2215-0366(18)30090-7

24. Mayoral-van Son J, de la Foz VO, Martinez-Garcia O, Moreno T, ParrillaEscobar M, Valdizan EM, et al. Clinical outcome after antipsychotic treatment discontinuation in functionally recovered first-episode nonaffective psychosis individuals: a 3-year naturalistic follow-up study. J Clin Psychiatry (2016) 77:492-500. doi: 10.4088/JCP.14m09540

25. Lezak MD. Neuropsychological Assessment. 3rd ed. New York, NY: Oxford University Press (1995).

26. Wechsler D. WAIS-III: Wechsler adult intelligence scale. In: Administration and Scoring Manual, 3rd edn. San Antonio, TX: Psychological Corporation (1997).

27. Yamashima T, Yoshida M, Kumahashi K, Matsui M, Koshino Y, Higashima M, et al. [The Japanese version of RBANS (Repeatable Battery for the Assessment of Neuropsychological Status)]. No To Shinkei (2002) 54:463-71.

28. Randolph C. Repeatable Battery for the Assessment of Neuropsychological Status. San Antonio, TX: Psychological Corporation (1998).

29. Frances A, Pincus HA, First MB. The global assessment of functioning scale (GAF). In: American Psychiatric Association. Diagnostic and Statistical Manual of Mental Disorders, 4th ed. Washington, DC: American Psychiatric Association (1994). p. 32.

30. Gold LH. DSM-5 and the assessment of functioning: the World Health Organization Disability Assessment Schedule 2.0 (WHODAS 2.0). J Am Acad Psychiatry Law (2014) 42:173-81.

31. Oliver JP. The social care directive: development of a quality of life profile for use in community services for the mentally ill. Soc Work Soc Sci Rev. (1991) 3:5-45.

32. van Nieuwenhuizen C, Schene AH, Koeter MW, Huxley PJ. The Lancashire quality of life profile: modification and psychometric evaluation. Soc Psychiatry Psychiatr Epidemiol. (2001) 36:36-44. doi: 10.1007/s0012700 50288

33. Wiersma D, DeJong A, Ormel J. The Groningen social disabilities schedule: development, relationship with I.C.I.D.H., and psychometric properties Int J Rehabil Res. (1988) 11:213-24. doi: 10.1097/00004356-19880900000001

34. Goldman HH, Skodol AE, Lave TR. Revising axis V for DSM-IV: a review of measures of social functioning. Am J Psychiatry (1992) 149:1148-56. doi: 10.1176/ajp.149.9.1148

35. American Psychiatric Association. Diagnostic and Statistical Manual of Mental Disorders. 4th ed. Washington, DC: American Psychiatric Association (2000).

36. Goodman SH, Sewell DR, Cooley EL, Leavitt N. Assessing levels of adaptive functioning: the role functioning scale. Commun. Ment Health J. (1993) 29:119-31. doi: 10.1007/BF00756338

37. Ware JE, Kosinski M. SF-36 Physical and Mental Health Summary Scales: a User's Manual. Boston, MA: The Health Institute, New England Medical Center (1994).

38. Gspandl S, Peirson RP, Nahhas RW, Skale TG, Lehrer DS. Comparing Global Assessment of Functioning (GAF) and World Health Organization Disability Assessment Schedule (WHODAS) 2.0 in schizophrenia. Psychiatry Res. (2018) 259:251-3. doi: 10.1016/j.psychres.2017.10.033

39. Undurraga J, Murru A, Vieta E. Early medication discontinuation on long-term recovery outcome in first-episode psychosis. JAMA 
Psychiatry (2014) 71:206-7. doi: 10.1001/jamapsychiatry.201 3.2993

40. Hui CL, Chen EY. Early medication discontinuation on long-term recovery outcome in first-episode psychosis. JAMA Psychiatry (2014) 71:207-8. doi: 10.1001/jamapsychiatry.2013.3697

41. Husa AP, Moilanen J, Murray GK, Marttila R, Haapea M, Rannikko I, et al. Lifetime antipsychotic medication and cognitive performance in schizophrenia at age 43 years in a general population birth cohort. Psychiatry Res. (2017) 247:130-8. doi: 10.1016/j.psychres.2016. 10.085

42. Gaebel W, Riesbeck M, Wölwer W, Klimke A, Eickhoff M, von Wilmsdorff $\mathrm{M}$, et al. Predictors for symptom re-exacerbation after targeted stepwise drug discontinuation in first-episode schizophrenia: results of the first-episode study within the German research network on schizophrenia. Schizophr Res. (2016) 170:168-76. doi: 10.1016/j.schres.2015.10.024
43. Alvarez-Jiménez M, Parker AG, Hetrick SE, McGorry PD, Gleeson JF. Preventing the second episode: a systematic review and meta-analysis of psychosocial and pharmacological trials in first-episode psychosis. Schizophr Bull. (2011) 37:619-30. doi: 10.1093/schbul/sbp129

Conflict of Interest Statement: The authors declare that the research was conducted in the absence of any commercial or financial relationships that could be construed as a potential conflict of interest.

Copyright (C) 2018 Omachi and Sumiyoshi. This is an open-access article distributed under the terms of the Creative Commons Attribution License (CC BY). The use, distribution or reproduction in other forums is permitted, provided the original author(s) and the copyright owner(s) are credited and that the original publication in this journal is cited, in accordance with accepted academic practice. No use, distribution or reproduction is permitted which does not comply with these terms. 